\title{
Simulation of the oil supply through the connecting rod to the piston cooling channels in medium speed engines
}

The importance of the oil flow simulation in connecting rod oil channels during the engine development process is recently increasing. This can be observed either in medium speed engines, where, as one of the traditional solutions, the oil for piston cooling is supplied through the connecting rod, or in automotive engine VCR (variable compression ratio) connecting rods, where engine oil is used to change the compression ratio of the engine. In both cases, precise numerical results are necessary to shorten the prototyping period and to reduce the overall development cost.

The multi-physics character of the simulation problem basically consists of the interaction between the dynamics of the crank train components and the oil flow. For the oil supply to the piston cooling channels through the connecting rod in medium speed engines, being the objective of this paper, a major influencing factor is the oil pressure behavior in the piston cooling gallery providing periodical interaction with its supply. At the same time, the connecting rod elastic deformation during engine operation can be regarded as negligible and the planar motion of the connecting rod can be reproduced by combination of translational and rotational acceleration fields in the CFD solver.

The paper includes the description of the applied simulation approach, the results and a comparison with the state-of-the art calculation without consideration of the above-mentioned influencing factors.

Key words: oil flow simulation, piston cooling, connecting rod dynamics, medium speed engines

\section{Introduction}

The thermal load of the pistons in medium speed engines is on a high level which requires proper cooling. Such cooling is typically realized by engine oil, which is provided to the piston cooling gallery transferring the heat from the piston internal surfaces. The oil flow rate utilized for piston cooling is therefore the major parameter to control piston temperature and the durability of the piston itself.

Simulating the oil supply to the piston through oil passages in the connecting rod and the piston pin represents a multi-physics problem. It basically consists of aspects of fluid mechanics which describe the flow through oil passages and the piston cooling gallery as well as of the dynamics of planar or reciprocating motions of the connecting rod and the piston and their influence on the oil flow.

Because of these various influence parameters, precise determination of the oil supply through the connecting rod for piston cooling during engine design is a very complex task. Hence, simplified engineering calculations or simulations are typically applied. Correctness of such estimations is subsequently verified by engine testing on the prototypes.

A precise simulative approach to solving such a problem includes e.g. coupling of 1D oil flow and multibody system (MBS) simulation of the connecting rod. This was recently done for the simulation of FEV's VCR connecting rod [1]. Such a simulation approach is necessary for a VCR connecting rod, where deformation of the connecting rod body influences the volume of the internal oil chambers and thus the effective connecting rod length as well as the switching process between the two compression ratios. It is however a sophisticated and time consuming approach.

For the simulation of the oil supply through the connecting rod to the piston cooling gallery in a medium speed engine with a conventional rigid connecting rod, a more time effective and straightforward methodology is required.
The optimum simulation approach according to the authors is a CFD simulation, which reproduces the effects of connecting rod movement by a combination of translational and rotational acceleration fields. This can be performed with enough precision, since the deformation of the connecting rod structure has only marginal influence on the oil supply to the piston via the connecting rod oil drillings. In parallel, the variable discharge pressure from the oil passages to the piston cooling gallery should be considered by coupled CFD simulation of the oil sloshing in the piston cooling gallery.

Such simulation has been recently performed during development of a new medium speed engine in the FEV Group, which is the basis of this paper.

\section{Modelling of connecting rod dynamics for CFD simulation of oil flow in the crank mechanism}

The kinematics and dynamics of a crank mechanism is historically a well-known topic. The beginnings of such considerations reach even back to the era of reciprocating steam machines, being the predecessor of today's internal combustion piston engines.

Regardless of the very simple principle of transferring the reciprocating movement of the piston into rotational movement of the crankshaft, the planar movement of the connecting rod is difficult to be precisely described by mathematical functions, which can be effectively applied either in order to understand the crank mechanism kinematics or for engineering calculations. Therefore, in the classic approach, the connecting rod is reduced to a two-point mass equivalent system, in which one realizes a translational motion (along with piston) and the second one, being attached to the crankpin, the rotational motion - Fig.1. 


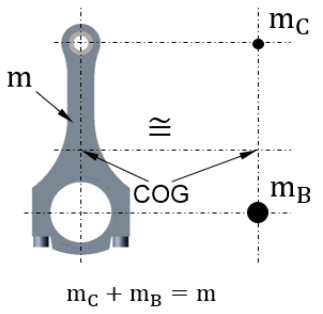

Fig. 1. Reduction of connecting rod to two-mass system [2,3]

For the oscillating mass of connecting rod, the acceleration measured along cylinder axis (with a positive value for the vector oriented from cylinder head to crankshaft) can be expressed by following equation:

$$
a_{C}=\omega^{2} r(\cos (\varphi)+\lambda \cos (2 \varphi))
$$

where: $\mathrm{a}_{\mathrm{C}}-$ acceleration of connecting rod oscillating mass, $\omega-$ crankshaft angular velocity, $r$ - crank throw radius, $\varphi-$ crankshaft angle, $\lambda$ - crank radius to connecting rod length ratio.

The centripetal acceleration $\mathrm{a}_{\mathrm{B}}$ of the rotating part can be expressed easily by equation (2):

$$
\mathrm{a}_{\mathrm{B}}=\omega^{2} \mathrm{r}
$$

The above-mentioned equations (1) and (2) are simplifications, not only due to reduction of connecting rod to twopoint mass system, but also due to the fact, that the motion of oscillating masses described by equation (1) takes into account only the first two elements of an infinite Tailor sequence.

Such simplified considerations can be found in nearly all textbooks related to basics of internal combustion engines (e.g. [2, 3], etc.). They are precise enough to understand or to calculate most of the effects of crank mechanism motion. These are e.g. time dependent changes of the cylinder volume during combustion and gas exchange simulations, piston and connecting rod mechanical loads, crankshaft balancing considerations, hydrodynamic bearing loads, torsional vibration excitations or bending loads of the crankshaft.

However, this simplified approach for determination of connecting rod movement is not sufficient for considerations where local mass forces acting along the connecting rod shank are of significance. Such effects are important for precise simulation of e.g. connecting rod buckling or the oil flow in the oil channels supplying engine oil from the big to small eye of the connecting rod.

For such considerations, the basics of rigid body mechanics for planar motion can be applied. Defining coordinate systems and basic locations acc. to Fig. 2, the acceleration of any point A can be described by equation 3 [4]:

$$
\overrightarrow{\mathrm{a}_{\mathrm{A}}}=\overrightarrow{\mathrm{a}_{\mathrm{B}}}+\overrightarrow{\mathrm{a}_{\mathrm{A} \mid \mathrm{B}}}{ }^{(\tau)}+{\overrightarrow{\mathrm{a}_{\mathrm{A} \mid \mathrm{B}}}}^{(\mathrm{n})}
$$

where: $\overrightarrow{a_{A}}-$ acceleration of any point $A$ of connecting rod, $\overrightarrow{a_{B}}-$ acceleration of point $B$ subjected to rotational motion, $\underset{\mathrm{a}_{\mathrm{A} \mid \mathrm{B}}}{\longrightarrow}(\tau)$ - tangential acceleration of any point $\mathrm{A}$ of connecting rod in relation to point $\mathrm{B}, \overrightarrow{\mathrm{a}}_{\mathrm{A} \mid \mathrm{B}}{ }^{(\mathrm{n})}-$ normal (centripetal) acceleration of any point $A$ of connecting rod in relation to point B.

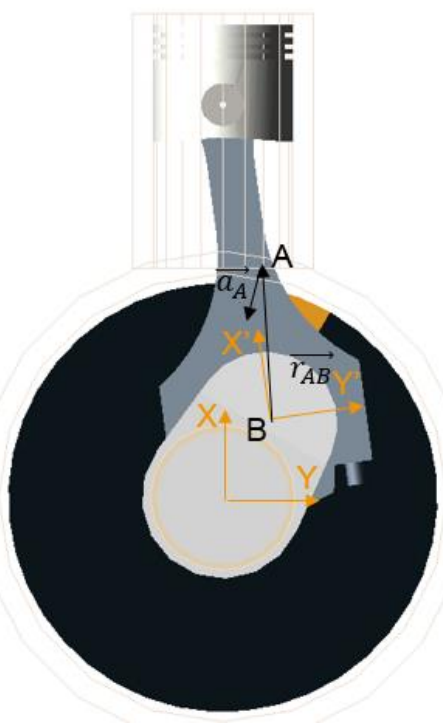

Fig. 2. Coordinate systems and basic locations in crank mechanisms

Equation (3) can be expressed by use of angular velocity $\overrightarrow{\omega_{\beta}}$ and angular acceleration $\overrightarrow{\varepsilon_{\beta}}$ of the connecting rod by the following relation:

$$
\overrightarrow{\mathrm{a}_{\mathrm{A}}}=\overrightarrow{\mathrm{a}_{\mathrm{B}}}+\overrightarrow{\varepsilon_{\beta}} \times \overrightarrow{\mathrm{r}_{\mathrm{AB}}}+\overrightarrow{\omega_{\beta}} \times\left(\overrightarrow{\omega_{\beta}} \times \overrightarrow{\mathrm{r}_{\mathrm{AB}}}\right)
$$

where $\overrightarrow{r_{A B}}$ is the vector of the position of point $A$ in relation to point $\mathrm{B}$.

Application of accelerations according to equation (4) expressed in the coordinate system $X^{\prime} Y^{\prime} Z$ ' allows to simulate the effect of connecting rod dynamics in the CFD solver. For such a case, the mesh of the connecting rod oil channels is stationary, and the flow volume is subjected to accelerations according to above-mentioned general equation.

In order to express the normal (centripetal) acceleration of point $\mathrm{B}$ in coordinate system $\mathrm{X}^{\prime} \mathrm{Y}^{\prime} \mathrm{Z}$ ', the effect of connecting rod tilting needs to be considered. The result of such considerations is:

$$
\overrightarrow{a_{B}}=r \omega^{2}\left[\begin{array}{c}
\lambda \sin ^{2}(\varphi)- \\
\left.\cos (\varphi) \sqrt{1-\lambda^{2} \sin ^{2}(\varphi)}\right) \\
\left(\begin{array}{c}
(-1) \lambda \sin (\varphi) \cos (\varphi)- \\
\sin (\varphi) \sqrt{1-\lambda^{2} \sin ^{2}(\varphi)}
\end{array}\right) \\
0
\end{array}\right]
$$

Taking into account that connecting rod angular velocity and acceleration are accordingly the first and the second time derivative of the connecting rod tilting angle, after simple considerations and mathematical transformations, they can be expressed in coordinate system $X^{\prime} Y^{\prime} Z$ ' as follows:

$$
\begin{aligned}
& \overrightarrow{\omega_{\beta}}=\left[\begin{array}{c}
0 \\
0 \\
\frac{(-1) \lambda \omega \cos (\varphi)}{\sqrt{1-\lambda^{2} \sin ^{2}(\varphi)}}
\end{array}\right] \\
& \overrightarrow{\varepsilon_{\beta}}=\left[\begin{array}{c}
0 \\
0 \\
\frac{\lambda \omega^{2} \sin (\varphi)-\frac{\lambda^{3} \omega^{2} \sin (\varphi) \cos ^{2}(\varphi)}{1-\lambda^{2} \sin ^{2}(\varphi)}}{\sqrt{1-\lambda^{2} \sin ^{2}(\varphi)}}
\end{array}\right]
\end{aligned}
$$


The above-mentioned approach was developed for the purpose of the CFD simulation presented in this paper. Since similar considerations were not found in the relevant literature, the simple multibody analysis was done with FEV's Virtual Dynamics simulation software in order to cross-check the theoretical considerations.

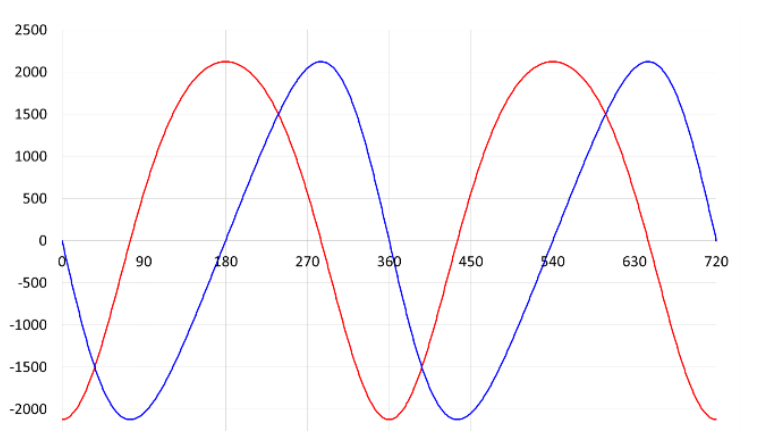

$-2500$

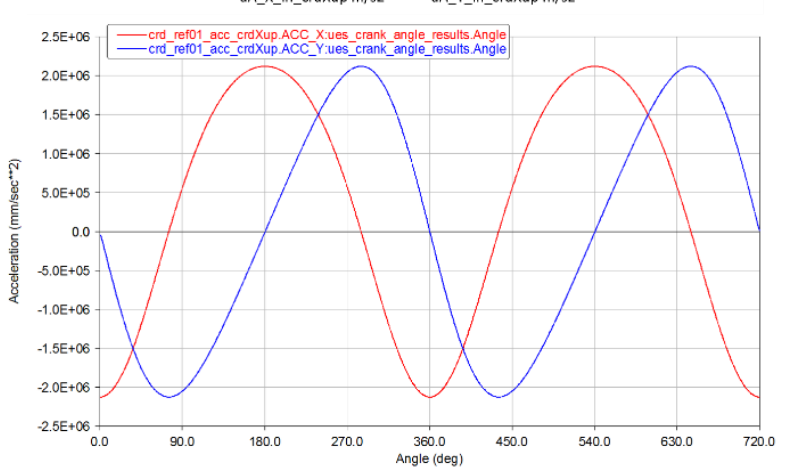

Fig. 3. Acceleration of the connecting rod's big eye center expressed in the connecting rod coordinate system calculated analytically by use of equations 4-7 (top) and simulated by use of MBS (bottom)
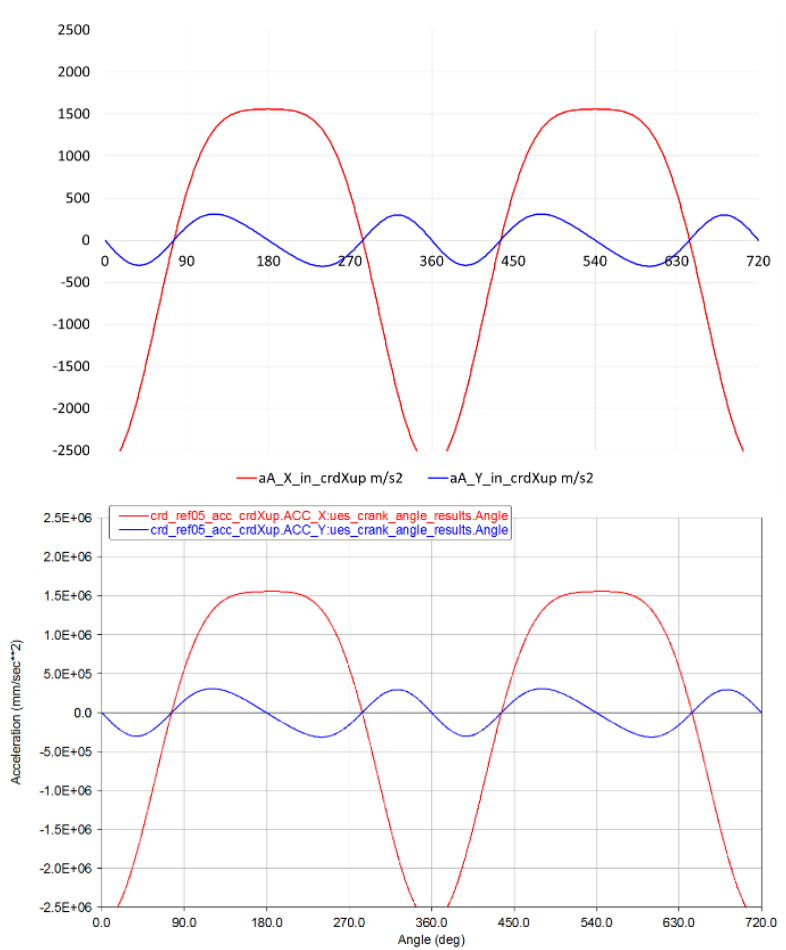

Fig. 4. Acceleration of the connecting rod's small eye center expressed in the connecting rod coordinate system calculated analytically by use of equations 4-7 (top) and simulated by use of MBS (bottom)
The acceleration was checked at the connecting rod's big eye center (Fig. 3), at the small eye center (Fig. 4) as well as at an arbitrarily selected point A (Fig. 5), and expressed in coordinate system X'Y'Z'. The position vector of point A relative to connecting rod length $L$ was defined as:

$$
\overrightarrow{\mathrm{r}_{\mathrm{AB}}}=\left[\begin{array}{c}
0.25 \mathrm{~L} \\
-0.05 \mathrm{~L} \\
0
\end{array}\right]
$$

In all three cases, the acceleration calculated analytically corresponds precisely with the results derived by the MBS simulation proving the correctness of the approach expressed by equations $4-7$.

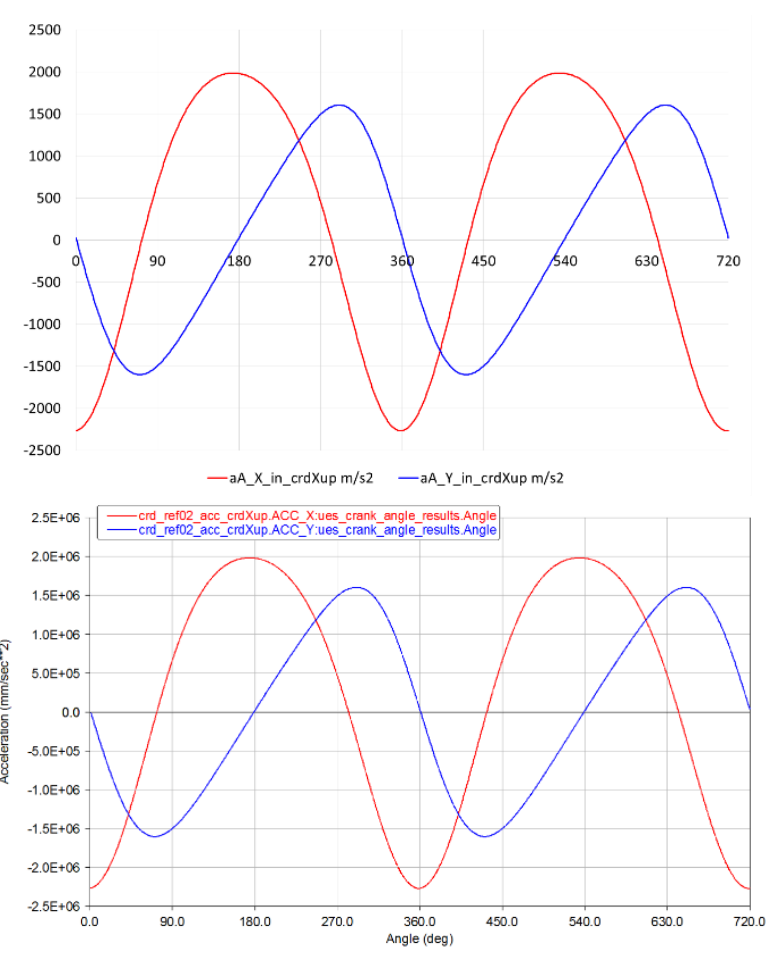

Fig. 5. Acceleration of connecting rod's point A (see text) expressed in the connecting rod coordinate system calculated analytically by use of equations 4-7 (top) and simulated by use of MBS (bottom)

\section{CFD modelling of the oil flow in the crank mechanism}

The precise determination of the oil flow through the connecting rod to the piston for cooling purposes and for optimization of the oil flow path by means of CFD simulation requires a properly determined modelling methodology. One of the key points of such a simulation approach is to use the exact geometry of the oil passages without any simplification which would influence the flow. The exemplary geometry used for the simulation, which results are presented in this paper, is shown in Fig. 6. It consists of the connecting rod oil drilling, a piston pin bearing groove as well as of piston pin and piston oil drillings.

The flow is described by transient Navier-Stokes and continuity conservation equations:

$$
\begin{gathered}
\rho \frac{\mathrm{Du}}{\mathrm{Dt}}=-\nabla p+\nabla * \tau+\rho \mathbf{g} \\
\frac{\partial \rho}{\partial \mathrm{t}}+\nabla *(\rho \mathbf{u})=0
\end{gathered}
$$


where: $\frac{\mathrm{D}}{\mathrm{Dt}}-$ material derivative, $\rho$ - density, $\mathbf{u}$ - velocity, $\nabla *$ - divergence, $\mathrm{p}$ - pressure, $\mathrm{t}-$ time, $\tau$ - stress tensor, $\mathrm{g}$ - body acceleration.

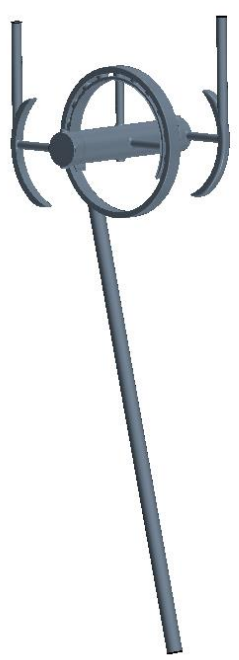

Fig. 6. Geometry used in the oil flow simulation

In the Navier-Stokes equation, the body acceleration factor was used to model the effect of connecting rod acceleration according to equations 4-7. The flow turbulence kinetic energy was calculated with a k- $\varepsilon$ turbulence model. The flow has been assumed to be isothermal. The time dependent part of the equations has been calculated with an implicit method. For the equations discretization, the $2^{\text {nd }}$ order upwind scheme was used. The SIMPLE algorithm was used for linking the Navier-Stokes and the continuity equation. The sets of discretized equations were solved using the Gauss-Siedel method.

In order to obtain reliable results, a fine quality mesh has been prepared. The numerical mesh consists of polyhedral elements. Prism layer has been included to properly model the boundary layer and, as a result, to predict pressure drop with high accuracy. Wall y+ values has been kept around 1. In the long oil drillings, the volume mesh has been obtained from a surface elements extrusion operation. With that, a more unfirmed mesh was generated.

For the inlet boundary condition, the stagnation pressure was used. The value of stagnation pressure was derived from a state-of-the-art 1-dimensional model of the engine lubrication system. The discharge was assumed to be a pressure boundary condition. The time dependent value of pressure at the model discharge was calculated in a separate CFD model for the piston cooling gallery, where the air-oil mixture is subjected to the acceleration resulting from the piston movement.

In order to conjugate both simulations, a couple of iterations has been performed. The mass flow is provided as an input from the CFD oil flow model to the oil sloshing model of the piston cooling gallery. In return, the information about average pressure at the end of the piston oil channel has been transported back to the oil prediction analysis. The coupling system is presented in the Fig. 7 below.

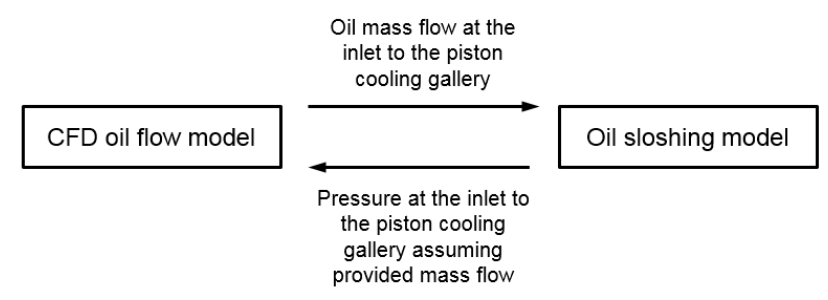

Fig. 7. Oil flow and oil sloshing models coupling

\subsection{Simulation of oil sloshing in the piston cooling gal- lery}

Oil sloshing simulation was performed separately using the Volume of Fluid (VOF) method, since the piston cooling gallery is only partially filled with oil. It describes the flow with transient Navier-Stokes equations including interaction between phases.

In this case, two fluids have been involved, one of them is engine oil, defined as a constant density liquid and the second is air, defined as ideal gas.

The inlet boundary condition applied in the model was the mass flow of oil flowing from the connecting rod bearing. It was exported from the simulation described in chapter 3 . The assumed discharge boundary was constant pressure, where oil from the gallery spills into the crankcase. Pressure deviation in the crankcase has been neglected. Influence of heat transfer through walls was neglected as well, considering the isothermal case. Analysis was run long enough to reach repeatable flow conditions in every rotation of the crankshaft.

Using this simulation, the transient pressure distribution on the oil inlet has been obtained. In order to detect air sucked from the oil gallery, the monitor was set in the drilling which supplies the oil to the gallery. As expected, air was mixing with oil and part of it was entering the inlet channel, but it was removed, when the flow reversed and oil flow into gallery was reestablished.

\section{Results}

The presented methodology has been applied to the simulation of the oil supply through the connecting rod to the piston cooling gallery in one of the medium speed engines developed by the FEV Group. For the CFD simulation, the commercial software Star-CCM+ has been used. The analysis has been performed for rated conditions, considering the geometry of oil passages shown in Fig. 6.

For the reference, a simulation without consideration of the crank mechanisms dynamics and the variable oil discharge pressure caused by oil sloshing in the piston cooling gallery has been performed. This reference simulation represents the state-of-the art approach, where it is assumed that effects of acceleration and deceleration occurring in the crank mechanism are compensating each other. By taking into account 5 bar of absolute total pressure at the connecting rod bearing and 1 bar (absolute static pressure) at the discharge on the piston cooling gallery, the calculated oil mass flow for the chosen example was on the level of $1.260 \mathrm{~kg} / \mathrm{s}$.

In order to determine the effects of crank mechanism dynamics and oil sloshing in the piston cooling gallery, additional two simulations have been performed, considering subsequently the above-mentioned aspects. 
4.1. Simulation considering crank mechanism dynamics and constant discharge pressure at the piston cooling gallery

For this simulation, the dynamics of the connecting rod was considered according to equations $4-7$, but the oil sloshing model was not taken into account. In the beginning of the simulation, the piston was in the top dead center position, and the simulation was run until the cycle-to-cycle results variation has decreased to a minimum. The resultant transient mass flow is shown in the Fig. 8.

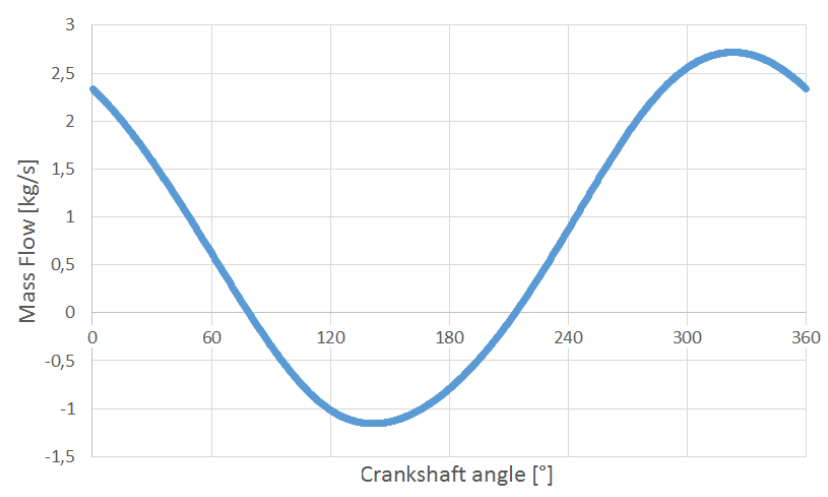

Fig. 8. Oil mass flow calculated with CFD model including the effect of connecting rod acceleration

The average mass flow is $0.765 \mathrm{~kg} / \mathrm{s}$ which is only $61 \%$ of the value calculated in the reference simulation. The explanation of such differences is the nonlinear behavior of the pressure losses. During the acceleration of the flow by the connecting rod movement these losses are significantly bigger than during deceleration, and they don't compensate each other over the complete engine cycle. Additionally, a significant backflow effect has been observed, which amount is however a matter of concern.

The oil backflow typically results in a pressure rise in the connecting rod bearing, which was not considered in the performed simulation. This pressure rise should result in a decrease of the amount of oil flowing back to the bearing. The precise determination of the backflow is possible by additional oil flow simulation inside the particular bearing which is planned to be performed in the near future.

Assuming that backflow doesn't exists at all, the simulated oil flow would be about $1.050 \mathrm{~kg} / \mathrm{s}$ being $84 \%$ of the oil flow calculated in the reference simulation.

\subsection{Simulation considering crank mechanism dynamics and the variable discharge pressure due to oil slosh- ing}

Using the mass flow presented in the previous chapter, the oil sloshing simulation has been performed. The base result is the transient value of the pressure at the boundary of the piston cooling gallery, which is effected by the oil-air mixture behavior. Other results refer to e.g. the piston crown cooling efficiency and the cooling gallery filling ratio. The oil flow CFD model has been updated with new discharge pressure boundary condition and run until the cycle-to-cycle results variation has decreased to minimum. Then, the simulations coupling has been introduced according to the scheme shown in Fig. 7. When both, pressure and mass flow values were repeatable, the solution has been considered to be converged. The pressure behavior during one cycle of crankshaft rotation is presented in Fig 9. Figure 10 shows the mass flow result of the converged simulation.

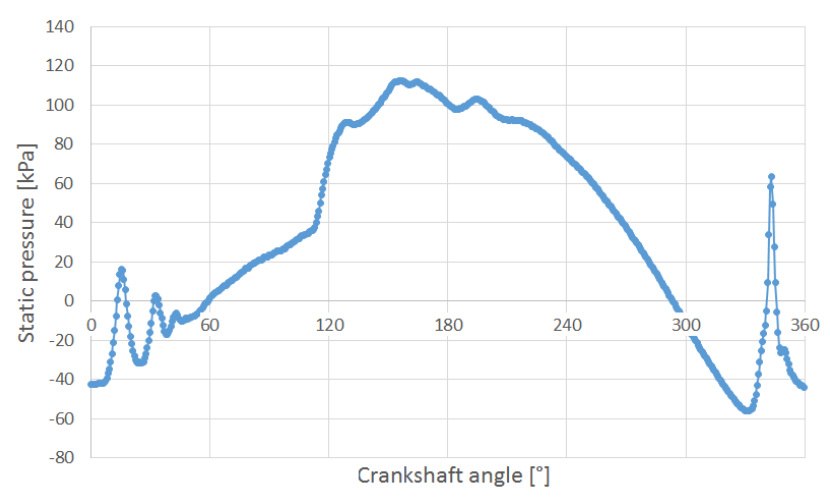

Fig. 9. Pressure behavior at the inlet to the piston cooling gallery

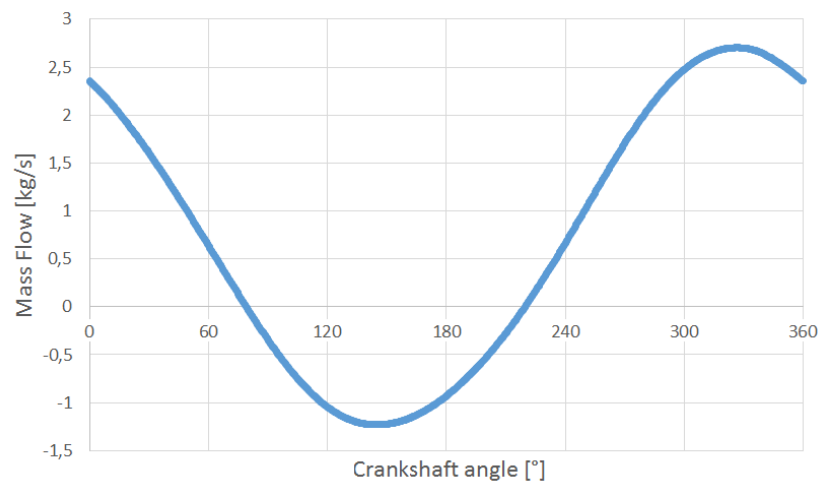

Fig. 10. Oil mass flow calculated with CFD model including the effect of connecting rod acceleration and the transient discharge pressure boundary conditions

The average value of mass flow is $0.697 \mathrm{~kg} / \mathrm{s}$, which means $9 \%$ of reduction in relation to the case, where the discharge pressure was considered to be constant.

\section{Summary}

The methodology for the simulation of the oil supply through the connecting rod to the piston cooling gallery in medium speed engines, being under development in the FEV Group, has been presented in this paper. Application of the advanced simulation enables to more precisely predict the piston cooling performance already in the early stages of an engine development process. A major point of the approach is consideration of the influence of connecting rod dynamics on the oil flow with a properly defined translational and rotation acceleration field integrated in the CFD simulation. Moreover, the simulation of the oil sloshing occurring in the piston cooling gallery and its influence on the discharge pressure from the oil supply passages has been introduced into the model.

The presented results show that due to the effect of connecting rod dynamics, the average value of oil mass flow is between 61 and $84 \%$ of the flow data determined without consideration of crank mechanism accelerations. The reduction by at least $16 \%$ is caused by a nonlinear behavior of 
pressure losses, which during the acceleration of the oil flow, caused by the connecting rod movement, are significantly higher than during deceleration. Due to this, a compensation of these effects doesn't occur over one complete engine cycle. Further reduction (up to additional 23\%) is caused by the oil backflow into the connecting rod bearing, which, in the performed simulation, is believed to be overestimated. In order to define the backflow more precisely, the oil flow behavior inside the crankpin bearing needs to be investigated. This is planned to be included in the simulation process in the near future.

Consideration of the variable discharge pressure in the oil supply passages of the crank mechanism showed a further $9 \%$ reduction of the resultant oil flow, which cannot be predicted by simplified calculation techniques.
The development of the simulation of the oil-air mixture in the piston cooling gallery additionally enables to predict more precisely such parameters as cooling gallery filling ratio, oil-air mixture backflow and piston cooling efficiency. All above-mentioned simulation approaches are essential to further improve the prediction of the piston temperature distribution and distortion.

\section{Acknowledgements}

Authors would like to thank M.Sc. Eng. Ladislav Kalina, MSc. Eng. Piotr Sułkowski (FEV Polska Sp. z o.o.), Dr.-Ing. Sven Lauer, Dr.-Ing. Carl Ritterskamp and Dipl.Ing. Ralf Rauschen (FEV Europe $\mathrm{GmbH}$ ) for their support and contribution in the development of the simulation approach presented in this paper.

\section{Nomenclature}

VCR Variable Compression Ratio

CFD Computational Fluid Dynamics

MBS Multibody Simulation

VOF Volume of Fluid (method)

\section{Bibliography}

[1] PENDOWSKI, D. Numerical methods to investigate the dynamic behavior of a VCR conrod. FEV Simulation and Calibration Day. Stuttgart 2018.

[2] JĘDRZEJOWSKI, J. Mechanika układów korbowych silników samochodowych, WKiE. Warszawa 1986.

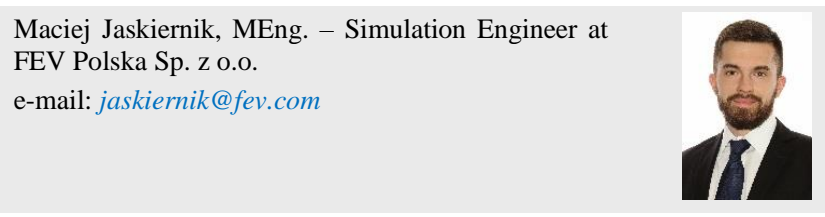

Jędrzej Walkowiak, MEng. - Simulation Engineer at FEV Polska Sp. z o.o.

e-mail:walkowiak@fev.com
[3] MAASS, H., KLIER, H. Die Verbrennungskraftmaschine, Band 2: Kräfte, Momente und deren Ausgleich in der Verbrennungskraftmaschine. Springer. 1981.

[4] LEYKO, J. Mechanika ogólna. Tom 1, tom 2. PWN. Warszawa 2002.
Konrad Buczek, MEng. - Deputy team leader of CAE department at FEV Polska Sp. z o.o.

e-mail: buczek@fev.com 